\title{
Reduction in first-order logic compared with reduction in implicational logic
}

\author{
Tigran M. Galoyan \\ Institute for Informatics and Automation Problems \\ National Academy of Sciences, Yerevan, Armenia \\ tigran.galoyan@gmail.com \\ Received by Greg Restall \\ Published November 9, 2007 \\ http://www.philosophy.unimelb.edu.au/ajl/2007 \\ (C) 2007 Tigran M. Galoyan
}

\begin{abstract}
In this paper we discuss strong normalization for natural deduction in the $\rightarrow \forall$ - fragment of first-order logic. The method of collapsing types is used to transfer the result (concerning strong normalization) from implicational logic to first-order logic. The result is improved by a complement, which states that the length of any reduction sequence of derivation term $r$ in first-order logic is equal to the length of the corresponding reduction sequence of its collapse term $r^{\mathrm{c}}$ in implicational logic.
\end{abstract}

Our basic logic calculus is the $\rightarrow \forall$ - fragment of minimal natural deduction for first-order logic over simply typed lambda-terms. This restriction regarding the minimal fragment does not mean a loss in general, since the full classical first-order logic can be embedded in this system by adding stability axiom. The method of collapsing types developed in [2] is used to get some results concerning the strong normalization of derivation terms in first-order logic.

\section{PRELIMINARIES}

Let us fix our language. Assume that we have a countable infinite set of function symbols $f, g, h \ldots$, and predicate symbols $P, Q, R \ldots$, each of arity $\geqslant 0$. Terms (object terms) $d, e, \ldots$, are defined inductively from object variables $x, y, z \ldots$, by the following rules:

I. object variable $x$ is a term,

2. if $\vec{d}$ is a list of terms and the arity of function symbol $f$ is the length of the list $\vec{d}$, then $f \vec{d}$ is a term, 
3. terms are defined only by rules I and 2 .

The set $F V(d)$ of free object variables of an object term $d$ is defined as usual.

Atomic formulas are $\perp$ (falsity) and $\mathrm{P} \overrightarrow{\mathrm{d}}$, where $\overrightarrow{\mathrm{d}}$ is a list of terms, $\mathrm{P}$ is a predicate symbol and the arity of $P$ is the length of $\vec{d}$.

Formulas are built from atomic formulas by implication $\varphi \rightarrow \psi$ and universal quantification $\forall x \varphi$.

Derivation terms $\mathrm{r}^{\varphi}, \mathrm{t}^{\varphi}, \mathrm{s}^{\varphi}, \mathrm{q}^{\varphi} \ldots$ (and also its set $\mathrm{FA}\left(\mathrm{r}^{\varphi}\right)$ of free assumption variables) are built from assumption variables $u^{\varphi}, v^{\varphi}, w^{\varphi} \ldots$ by the introduction and elimination rules for $\rightarrow$ and $\forall$ :

$\varphi-\mathfrak{u}^{\varphi}$ is a derivation term with $F A\left(\mathfrak{u}^{\varphi}\right)=\left\{\mathfrak{u}^{\varphi}\right\}$;

$\rightarrow^{+}-$implication introduction - if $r^{\psi}$ is a derivation term, then $\left(\lambda u^{\varphi} r^{\psi}\right)^{\varphi \rightarrow \psi}$ is a derivation term with $\mathrm{FA}\left(\left(\lambda u^{\varphi} r^{\psi}\right)\right)=\mathrm{FA}\left(r^{\psi}\right) \backslash\left\{u^{\varphi}\right\}$;

$\rightarrow^{-}$- implication elimination - if $\mathrm{t}^{\varphi \rightarrow \psi}$ and $s^{\varphi}$ are derivation terms, then $\left(t^{\varphi \rightarrow \psi} s^{\varphi}\right)^{\psi}$ is a derivation term with $F A\left(t^{\varphi \rightarrow \psi} s^{\varphi}\right)=F A\left(t^{\varphi \rightarrow \psi}\right) \cup$ $\mathrm{FA}\left(\mathrm{s}^{\varphi}\right)$;

$\forall^{+}-$universal quantification introduction - if $r^{\varphi}$ is a derivation term and $x$ is an object variable which satisfies the condition $x \notin \cup\left\{F V(\psi) \mid u^{\psi} \in\right.$ $\left.\mathrm{FA}\left(\mathrm{r}^{\varphi}\right)\right\}$, then $\left(\lambda x r^{\varphi}\right)^{\forall x \varphi}$ is a derivation term with $\mathrm{FA}\left(\lambda x \mathrm{r}^{\varphi}\right)=\mathrm{FA}\left(\mathrm{r}^{\varphi}\right)$;

$\forall^{-}$- universal quantification elimination - if $\mathrm{t}^{\forall x \varphi}$ is a derivation term and $\mathrm{d}$ is an object term, then $\left(t^{\forall x \varphi} d\right)^{\varphi_{x}[d]}$ is a derivation term with $F A\left(t^{\forall x \varphi} d\right)$ $=\mathrm{FA}\left(\mathrm{t}^{\forall x \varphi}\right)$.

We write $r^{\varphi}\left[u_{1}^{\psi_{1}}, \ldots, u_{m}^{\psi_{m}}\right]$ to indicate that the assumption variables free in $r^{\varphi}$ are in the list $u_{1}^{\psi_{1}}, \ldots, u_{m}^{\psi_{m}}$. We also use the notation $r: \varphi$ instead of $r^{\varphi}$.

DEFINITION I A formula $\varphi$ is called derivable from assumptions $\psi_{1}, \ldots, \psi_{m}$, if there is a derivation term $r^{\varphi}\left[u_{1}^{\psi_{1}}, \ldots, u_{m}^{\psi_{m}}\right]$ with different assumption variables $u_{1}^{\psi_{1}}, \ldots, u_{m}^{\psi_{m}}$.

In the case of classical logic: for any predicate symbol $\mathrm{P}$ the term $\mathrm{stab}_{\mathrm{P}}$ : $\forall \vec{x} . \neg \neg P \vec{x} \rightarrow P \vec{x}$ is a derivation term.

From now on we will use the word term for derivation terms (until there is no confusion with the notion of object terms) and type for formulas.

As we have mentioned the $\rightarrow \forall$ - fragment of minimal logic contains full classical first-order logic. As in [I] (Subsections I.I and 2.I) this can be seen as follows: 
I. Associate with any formula $\varphi$ in the language of classical first-order logic a finite list $\vec{\varphi}$ of formulas in our $\rightarrow \forall$ - fragment, by induction on $\varphi$ :

$$
\begin{aligned}
\mathrm{P} \overrightarrow{\mathrm{d}} & \mapsto \mathrm{P} \overrightarrow{\mathrm{d}} \\
\neg \varphi & \mapsto \vec{\varphi} \rightarrow \perp \\
\varphi \rightarrow \psi & \mapsto \vec{\varphi} \rightarrow \psi_{1}, \ldots, \vec{\varphi} \rightarrow \psi_{\mathrm{n}} \\
\varphi \wedge \psi & \mapsto \vec{\varphi}, \vec{\psi} \\
\varphi \vee \psi & \mapsto(\vec{\varphi} \rightarrow \perp),(\vec{\psi} \rightarrow \perp) \rightarrow \perp \\
\forall x \varphi & \mapsto \forall x \varphi_{1}, \ldots, \forall x \varphi_{\mathrm{m}} \\
\exists x \varphi & \mapsto \forall x(\vec{\varphi} \rightarrow \perp) \rightarrow \perp
\end{aligned}
$$

where we write $\vec{\varphi} \rightarrow \psi$ for $\left(\varphi_{1} \rightarrow\left(\varphi_{2} \rightarrow \cdots\left(\varphi_{m} \rightarrow \psi\right) \cdots\right)\right)$.

2. In any model $\mathcal{M}$, where $\perp$ is interpreted by falsity, we clearly have that a formula $\varphi$ in the language of full first-order logic holds under an assignment $\alpha$ iff all formulas in the assigned sequence $\vec{\varphi}$ hold under $\alpha$ (in our $\rightarrow \forall$ - fragment of minimal logic).

3. Our derivation calculus for the $\rightarrow \forall$ - fragment is complete in the following sense:

a formula $\varphi$ is derivable from stability assumptions $\forall \vec{x} . \neg \neg P \vec{x} \rightarrow P \vec{x}$ for all predicate symbols $P$ in $\varphi$ iff $\varphi$ is valid in any model under any assignment.

\section{STRONG NORMALIZATION}

It was shown in [I] that for pure implicational logic any term can be reduced to a normal form (w.r.t. $\rightarrow_{1}$ conversion, the one step reduction using $\beta$-conversion rule) and this form is uniquely determined. Moreover, it was shown that any reduction sequence terminates, i.e. any term is strongly normalizable. A derivation term is said to be in normal form if it is impossible to perform a reduction. Here we use the method of collapsing types (ref [2]) to transfer the result (concerning strong normalization, obtained in [I]) from implicational logic to first-order logic.

It must be mentioned that the general $\beta$-conversion rule is extended to first-order logic. In particular, we have

$$
\left(\lambda u^{\varphi} t^{\psi}\right) s^{\varphi} \text { converts into }\left(\rightarrow_{1}\right) t_{u}[
$$

where $t, s$ are derivation terms, $u$ is an assumption variable; and

$$
\left(\lambda \times r^{\varphi}\right) d \text { converts into }\left(\rightarrow_{1}\right) r^{\varphi_{x}[d]}
$$

where $x$ is an object variable, $d$ is an object term and $r$ is a derivation term.

For any formula $\varphi$ of first-order logic we define its collapse $\varphi^{c}$ by 


$$
\begin{aligned}
(\mathrm{P} \overrightarrow{\mathrm{d}})^{\mathrm{c}} & \equiv \mathrm{P} \\
(\varphi \rightarrow \psi)^{\mathrm{c}} & \equiv \varphi^{\mathrm{c}} \rightarrow \psi^{\mathrm{c}} \\
(\forall \times \varphi)^{\mathrm{c}} & \equiv \mathrm{T} \rightarrow \psi^{\mathrm{c}}
\end{aligned}
$$

where $T: \equiv \perp \rightarrow \perp$ (i.e. $T$ means tautology). Though, $\perp$ is an atomic formula it behaves like predicate symbols, i.e. $(\perp)^{\mathrm{c}} \equiv \perp$, therefore $(T)^{\mathrm{c}} \equiv T$.

For any derivation term $r^{\psi}$ in first-order logic we can now define its collapse $\left(r^{\psi}\right)^{c}$. It is obvious from this definition that for any derivation term $r^{\psi}$ in firstorder logic with free assumption variables $u_{1}^{\varphi_{1}}, \ldots, u_{m}^{\varphi_{m}}$ the collapse $\left(r^{\psi}\right)^{c}$ is a derivation term $\left(r^{c}\right)^{\varphi^{c}}$ in implicational logic with free assumption variables $u_{1}^{\varphi_{1}^{c}}, \ldots, u_{m}^{\varphi_{m}^{c}}$.

$$
\begin{aligned}
& \left(u^{\varphi}\right)^{\mathrm{c}} \equiv u^{\varphi^{\mathrm{c}}} \quad\left(\mathrm{c}_{4}\right) \\
& \left(\lambda u^{\varphi} r\right)^{c} \equiv \lambda u^{\varphi^{c}} r^{c} \quad \text { (c5) } \\
& \left(\mathrm{t}^{\varphi \rightarrow \psi} \mathrm{s}^{\varphi}\right)^{\mathrm{c}} \equiv \mathrm{t}^{\mathrm{c}} \mathrm{s}^{\mathrm{c}} \\
& (\lambda \times r)^{c} \equiv \lambda u^{\top} r^{c} \quad(c 7) \\
& \left(\mathrm{t}^{\forall \times \varphi} \mathrm{d}\right)^{\mathrm{c}} \equiv \mathrm{t}^{\mathrm{c}}\left(\lambda z^{\perp} z^{\perp}\right)^{\top} \quad \text { (c8) }
\end{aligned}
$$

Note that for any derivation term $r^{\psi}$, assumption variable $u^{\varphi}$ and derivation term $s^{\varphi}$ we have that $r^{\mathcal{C}}\left[s^{c}\right]$ is a derivation term in implicational logic (where the substitution of $s^{c}$ is done for the assumption variable $u^{\varphi^{c}}$ ), which is the collapse of $r[s]$. Also for any derivation term $r^{\psi}$, object variable $x$ and object term $d$ we have that $r_{x}[d]$ is a derivation term of $\psi_{x}[d]$ with collapse $\left(r_{x}[d]\right)^{c} \equiv r^{c}$.

LEMMA I If $\mathrm{r} \rightarrow 1 \mathrm{r}^{\prime}$ in first-order logic, then $\mathrm{r}^{\mathrm{c}} \rightarrow_{1}\left(\mathrm{r}^{\prime}\right)^{\mathrm{c}}$ in implicational logic ([I] - Subsection 2.2).

From Lemma I and the theorem, which states that any term in implicational logic is strongly normalizable, the following main result was obtained in [I]:

THEOREM 2 Any derivation term $\mathrm{r}$ in first-order logic is strongly normalizable.

Indeed, since the collapse $r^{c}$ of the term $r$ is a term in implicational logic and any term in implicational logic is strongly normalizable, i.e. any reduction sequence starting from $r^{c}$ terminates, then from Lemma I we conclude that any reduction sequence starting from $r$ also terminates, hence $r$ is strongly normalizable. But it is still conceivable that $r$ terminates (in terms of reduction sequence) before $r^{c}$, i.e. the reduction sequence of $r^{c}$ as defined is longer than the reduction sequence of $r$ that we chose. Our aim is to show that it is impossible, and both of the terms do the same number of one-step reductions.

First of all it is necessary to emphasize that it is not so obvious, since there is no bijective correspondence between a derivation term in first-order logic and its collapse. 
NOTE. Although, to any derivation term in first-order logic we identically associate collapse, it is not necessary for the converse to be true. The following instances illustrate this fact.

EXAMPLe I. Assume the collapse is $t^{c}=\lambda u^{\top} r^{c}$. Then there are two possible forms of derivation term $t$ (ambiguity):

I. on the one hand, since $T^{c} \equiv \top$, then $t^{c}=\lambda u^{\top} r^{c}=\lambda u^{T^{c}} r^{c}=\left(\lambda u^{\top} r\right)^{c}$ according to (c5); so, $t=\lambda u^{\top} r$;

2. on the other hand, $t^{c}=\lambda u^{\top} r^{c}=\left(\lambda x_{o b j} r\right)^{c}$ according to (c7); so, $t=$ $\lambda x_{o b j}$ r.

We write $x_{o b j}$ instead of $x$ to indicate the fact that $x$ is an object variable. This notion is extended on object terms too, e.g. $d_{o b j}$ instead of d. For the convenience, sometimes the obj pattern will be omitted, but implied.

EXAMPle 2. Assume $\varphi^{c}=T \rightarrow P$, where $P$ is any predicate symbol. Then there are two possible forms of a formula $\varphi$ (ambiguity):

I. on the one hand, since $(\perp \rightarrow \perp)^{\mathrm{c}}=T^{\mathrm{c}} \equiv \top=(\perp \rightarrow \perp)$, then $\varphi^{\mathrm{c}}=\top \rightarrow$ $\mathrm{P}=(\perp \rightarrow \perp) \rightarrow \mathrm{P}=(\perp \rightarrow \perp)^{\mathrm{c}} \rightarrow \mathrm{P}^{\mathrm{c}}=((\perp \rightarrow \perp) \rightarrow \mathrm{P})^{\mathrm{c}}$ according to (cI) and (c2); so, $\varphi=(\perp \rightarrow \perp) \rightarrow \mathrm{P}=\top \rightarrow \mathrm{P}$;

2. on the other hand, $\varphi^{\mathrm{c}}=\top \rightarrow \mathrm{P}=\top \rightarrow \mathrm{P}^{\mathrm{c}}=\left(\forall x_{\mathrm{obj}} \mathrm{P}\right)^{\mathrm{c}}$ according to (c3); so, $\varphi=\forall x_{\text {obj }}$ P.

Now we reformulate Theorem 2:

THEOREM 3 Any derivation term $\mathrm{r}$ in first-order logic is strongly normalizable. Moreover, for any reduction sequence $r=r_{1} \rightarrow_{1} \cdots \rightarrow_{1} r_{n}=r^{\prime}$ of a derivation term $r$ with normal form $r^{\prime}$, the length is identical to the length of the reduction sequence $r^{c}=r_{1}^{c} \rightarrow_{1} \cdots \rightarrow{ }_{1} r_{n}^{c}=\left(r^{\prime}\right)^{c}$ in implicational logic and $\left(r^{\prime}\right)^{c}$ is the normal form of $r^{c}$.

Proof: The first part of the theorem is plain due to Theorem 2. From Lemma I it simply follows that if $r^{c}$ terminates, so does $r$. It remains to prove that if $r$ terminates, so does $r^{c}$, i.e. $r^{c}$ terminates as soon as $r$. Assume that $r \rightarrow^{*} r^{\prime}$ and $r^{\prime}$ is the normal form of $r$; that is $r$ terminates and the last term of normalization reduction sequence is $r^{\prime}$. Here $\rightarrow^{*}$ denotes transitive and reflexive closure of $\rightarrow 1$. From Lemma I we obtain that $r^{c} \rightarrow^{*}\left(r^{\prime}\right)^{c}$ as well. Now it should be proved that $\left(r^{\prime}\right)^{c}$ cannot be normalized further, i.e. it terminates.

Let us suppose the opposite and come to contradiction. It means that there exists a term $r_{c}^{\prime \prime}$ such that $\left(r^{\prime}\right)^{c} \rightarrow_{1} r_{c}^{\prime \prime}$. So we have the next structure-view:

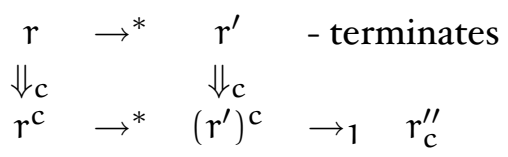


Therefore, we conclude that $\left(\mathrm{r}^{\prime}\right)^{\mathrm{c}}$ has a form

$$
\left(r^{\prime}\right)^{c}=t_{c}^{L}\left(\left(\lambda u_{c} t_{c}\right) s_{c}\right) t_{c}^{R}
$$

hence

$$
r_{c}^{\prime \prime}=t_{c}^{L}\left(t_{c_{u}}\left[s_{c}\right]\right) t_{c}^{R}
$$

where $t_{c}^{L}$ and $t_{c}^{R}$ are arbitrary terms and may be empty.

Let us denote by $t_{c}^{M}$ the middle part of $\left(r^{\prime}\right)^{c}$

$$
t_{c}^{M} \equiv\left(\lambda u_{c} t_{c}\right) s_{c} .
$$

More exactly $\left(r^{\prime}\right)^{c}$ has one of the two following forms:

(A) $\left[t_{c}^{L}\left(\left(\lambda u_{c} t_{c}\right) s_{c}\right)\right] t_{c}^{R}=\left(t_{c}^{L} t_{c}^{M}\right) t_{c}^{R}$,

(в) $t_{c}^{L}\left[\left(\left(\lambda u_{c} t_{c}\right) s_{c}\right) t_{c}^{R}\right]=t_{c}^{L}\left(t_{c}^{M} t_{c}^{R}\right)$.

REMARK. By $\tau(s)$ we denote the type of derivation term s, e.g. $\tau\left(s^{\varphi \rightarrow \psi}\right)=$ $\varphi \rightarrow \psi$. Let us consider the term $\mathrm{t}^{\mathrm{c}}\left(\left(\lambda z^{\perp} z^{\perp}\right)^{\top}\right)^{\mathrm{c}}$ in case when $\tau\left(\mathrm{t}^{\mathrm{c}}\right)=\top \rightarrow$ $\varphi^{c}=\top^{c} \rightarrow \varphi^{c}$. It is obvious that $\left(\left(\lambda z^{\perp} z^{\perp}\right)^{\top}\right)^{c}=\left(\lambda z^{\perp} z^{\perp}\right)^{\top}$. According to (c6) and (c8) there are two possible forms of term $r$ which collapse is $r^{c}=$ $\mathrm{t}^{\mathrm{c}}\left(\left(\lambda z^{\perp} z^{\perp}\right)^{\top}\right)^{\mathrm{c}}$ :

I. on the one hand $r=t^{\top \rightarrow \varphi}\left(\lambda z^{\perp} z^{\perp}\right)^{\top}$;

2. on the other hand $r=t^{\forall x_{o b j} \varphi} d_{o b j}$.

Inter alia, this remark can be viewed as one more example, which shows the accuracy of the note about inverse problem mentioned above.

We now consider the two forms of $\left(\mathrm{r}^{\prime}\right)^{\mathrm{c}}$ :

FOR THE FORM (A):

$$
\left(r^{\prime}\right)^{c}=\left(t_{c}^{L} t_{c}^{M}\right) t_{c}^{R}
$$

We consider two cases depending on the form of $t_{c}^{R}$.

$\operatorname{CASE}(\mathrm{A}-\mathrm{I}) . \mathrm{t}_{\mathrm{c}}^{\mathrm{R}}=\left(\lambda z^{\perp} z^{\perp}\right)^{\top}=\left(\left(\lambda z^{\perp} z^{\perp}\right)^{\top}\right)^{\mathrm{c}}$.

Let us denote: $\mathrm{q}^{\mathrm{c}} \equiv \mathrm{t}_{\mathrm{c}}^{\mathrm{L}}\left(\left(\lambda \mathrm{u}_{\mathrm{c}} \mathrm{t}_{\mathrm{c}}\right) \mathrm{s}_{\mathrm{c}}\right)$, hence $\left(\mathrm{r}^{\prime}\right)^{\mathrm{c}}=\mathrm{q}^{\mathrm{c}}\left(\left(\lambda z^{\perp} z^{\perp}\right)^{\top}\right)^{\mathrm{c}}$.

From the remark mentioned above we obtain that either

$$
\mathrm{r}^{\prime}=\mathrm{q}^{\top \rightarrow \varphi}\left(\lambda z^{\perp} z^{\perp}\right)^{\top}
$$

or

$$
\mathrm{r}^{\prime}=\mathrm{q}^{\forall x \varphi} \mathrm{d}
$$


$\operatorname{CASE}(\mathrm{A}-\mathrm{I}-\mathrm{I}) \cdot \mathrm{r}^{\prime}=\mathrm{q}^{\top \rightarrow \varphi}\left(\lambda z^{\perp} z^{\perp}\right)^{\top}$ and $\mathrm{q}^{\mathrm{c}}=\mathrm{t}_{\mathrm{c}}^{\mathrm{L}}\left(\left(\lambda \mathrm{u}_{\mathrm{c}} \mathrm{t}_{\mathrm{c}}\right) \mathrm{s}_{\mathrm{c}}\right)=\mathrm{t}_{\mathrm{c}}^{\mathrm{L}} \mathrm{t}_{\mathrm{c}}^{\mathrm{M}}$ and $\tau\left(\mathbf{q}^{\mathfrak{c}}\right)=\top \rightarrow \varphi^{\mathfrak{c}}$.

Since $t_{c}^{M}=\left(\lambda u_{c} t_{c}\right) s_{c} \neq\left(\lambda z^{\perp} z^{\perp}\right)^{\top}$ then according to (c4)-(c8) we conclude that there is only one possible form for $q^{c}$, that is-(c6). It follows that $\exists t_{L}, t_{M}$ terms, which satisfy these equations: $t_{c}^{L}=\left(t_{L}\right)^{c}$ and $t_{c}^{M}=\left(t_{M}\right)^{c}$, hence $q^{c}=\left(t_{L}\right)^{c}\left(t_{M}\right)^{c}$. Let us denote: $\left(t_{M L}\right)^{c} \equiv \lambda u_{c} t_{c}$, so we have $\left(t_{M}\right)^{c}=\left(\lambda u_{c} t_{c}\right) s_{c}=\left(t_{M L}\right)^{c} s_{c}$. Depending on the form $s_{c}\left(=\left(\lambda z^{\perp} z^{\perp}\right)^{\top}\right.$ or not) we get either $t_{M}=t_{M L}^{\forall x \psi} s$, where $s=e_{o b j}$, or $t_{M}=t_{M L}^{\psi \rightarrow \omega} s$, where $s^{c}=s_{c}$ ( $s_{c}$ is a derivation term). As we have $\left(t_{M L}\right)^{c} \equiv \lambda u_{c} t_{c}$, then according to (c5) and (c 7$)$ there are two possible forms of term $t_{M L}$ which collapse is $\lambda u_{c} t_{c}: t_{M L}=\lambda x_{o b j} t$, if $\tau\left(u_{c}\right)=\top$ or $t_{M L}=\lambda u t$, if $\tau\left(u_{c}\right) \neq \top$, where $u^{c}=u_{c}$ and $t^{c}=t_{c}$. Therefore, $t_{c}^{M}=\left(t_{M}\right)^{c}=\left[\left(\lambda x_{o b j} t\right) e_{o b j}\right]^{c}$ or $t_{c}^{M}=\left(t_{M}\right)^{c}=$ $[(\lambda u t) s]^{c}$, which means that in both cases the term $r^{\prime}$ contains subterm $\left(\lambda x_{o b j} t\right) e_{o b j}$ or $(\lambda u t) s$ respectively, i.e. we could have performed one more $\rightarrow 1$ reduction for $\mathrm{r}^{\prime}$, which contradicts our theorem condition that $r^{\prime}$ terminates.

$\operatorname{CASE}(A-I-2) \cdot r^{\prime}=q^{\forall x \varphi} d$ and $q^{c}=t_{c}^{L}\left(\left(\lambda u_{c} t_{c}\right) s_{c}\right)=t_{c}^{L} t_{c}^{M}$ and $\tau\left(q^{c}\right)=$ $\top \rightarrow \varphi^{c}$. This case is similar to the case (a-I-I).

$\operatorname{CASE}(\mathrm{A}-2) . \mathrm{t}_{\mathrm{c}}^{\mathrm{R}} \neq\left(\lambda z^{\perp} z^{\perp}\right)^{\top}$.

According to (c4)-(c8) we conclude that there is only one possible form for $\left(r^{\prime}\right)^{c}$, that is (c6): it follows that $r^{\prime}=q^{\varphi \rightarrow \psi_{t}} t_{R}$, where $\left(t_{R}\right)^{c}=t_{c}^{R}$ and $q^{c}=t_{c}^{L} t_{c}^{M}$, hence we come to the case (a-I-I) when $\tau\left(q^{c}\right)=\varphi^{c} \rightarrow \psi^{c}$.

FOR THE FORM (B):

$$
\left(\mathrm{r}^{\prime}\right)^{\mathrm{c}}=\mathrm{t}_{\mathrm{c}}^{\mathrm{L}}\left(\mathrm{t}_{\mathrm{c}}^{\mathrm{M}} \mathrm{t}_{\mathrm{c}}^{\mathrm{R}}\right)
$$

Since $t_{c}^{M} t_{c}^{R}$ does not have the form $\left(\lambda z^{\perp} z^{\perp}\right)^{\top}$, it follows that according to (c4)-(c8) there is only one possible form for $\left(r^{\prime}\right)^{c}$, that is (c6). Hence, $r^{\prime}=t_{L} q$, where $\left(t_{L}\right)^{c}=t_{c}^{L}$ and $q^{c}=t_{c}^{M} t_{c}^{R}=\left(\left(\lambda u_{c} t_{c}\right) s_{c}\right) t_{c}^{R}$. According to $\left(c_{4}\right)-(c 8) q^{c}$ may have one of the two following forms: (c6) or (c8). Depending on the form of $t_{c}^{R}\left(=\left(\lambda z^{\perp} z^{\perp}\right)^{\top}\right.$ or not) we get either $q=t_{M} d_{o b j}$, where $\left(t_{M}\right)^{c}=t_{c}^{M}$ or $q=t_{M} t_{R}$, where $\left(t_{M}\right)^{c}=t_{c}^{M}$ and $\left(t_{R}\right)^{c}=t_{c}^{R}$ respectively. In both cases we have $t_{M}$ which satisfies the equation $\left(t_{M}\right)^{c}=\left(\lambda u_{c} t_{c}\right) s_{c}$. The rest is similar to the case (a-I-I).

All the cases have been considered, hence the theorem is proved by the methods of contradiction.

Using the last result (Theorem 3) the upper bound for the length of arbitrary reduction sequences obtained in [3]. (obtained only for implicational logic) can be extended to include first-order logic. So we obtain, that in first-order logic any reduction sequence for a term $r$ is bounded by

$$
2_{g\left(r^{c}\right)}\left(l\left(r^{c}\right)\right)
$$


where $r^{c}$ is the collapse of the term $r, l\left(r^{c}\right)$ and $g\left(r^{c}\right)$ denote the length and degree of the term $r^{c}$ respectively. Here $2_{m}(n)$ is recursively defined by $2_{0}(n)=n$ and $2_{m+1}(n)=2^{2 m(n)}$.

\section{REFERENCES}

[I] H. Schwichtenberg. Normalization. Mathematisches Institut, Universitat Munchen, Germany 1989.

[2] A. Troelstra and D. van Dalen Constructivism in mathematics. An introduction. Studies in Logic and the Foundations of Mathematics, Vol. I2I, I23 Amsterdam: North Holland 1988

[3] A. Beckman Exact bounds for lengths of reductions in typed $\lambda$-calculus. Institut für mathematische Logik und Grundlagenforschung der Westfälischen Wilhelms-Universität Münster Einsteinstr. 62, D-48I49 Münster, Germany 2003 
The Australasian fournal of Logic (ISSN I448-5052) disseminates articles that significantly advance the study of logic, in its mathematical, philosophical or computational guises. The scope of the journal includes all areas of logic, both pure and applied to topics in philosophy, mathematics, computation, linguistics and the other sciences.

Articles appearing in the journal have been carefully and critically refereed under the responsibility of members of the Editorial Board. Only papers judged to be both significant and excellent are accepted for publication.

The journal is freely available at the journal website at

$$
\text { http://www.philosophy.unimelb.edu.au/ajl/ }
$$

All issues of the journal are archived electronically at the journal website.

SuBSCRIPTIONS Individuals may subscribe to the journal by sending an email, including a full name, an institutional affiliation and an email address to the managing editor at ajl-editors@unimelb.edu.au Subscribers will receive email abstracts of accepted papers to an address of their choice. For institutional subscription, please email the managing editor at ajl-editors@unimelb.edu.au.

Complete published papers may be downloaded at the journal's website at http: //www.philosophy.unimelb.edu.au/ajl/ The journal currently publishes in pdf format.

Submission The journal accepts submissions of papers electronically. To submit an article for publication, send the $\mathrm{LT}_{\mathrm{E}} \mathrm{X}$ source of a submission to a member of the editorial board. For a current list of the editorial board, consult the website.

The copyright of each article remains with the author or authors of that article. 